\title{
Integrative and collaborative care models between pediatric oral health and primary care providers: a scoping review of the literature
}

\author{
Tylor L. Gauger, DDS ${ }^{1}$; Lisa A. Prosser, PhD, MS ${ }^{2,3}$; Margherita Fontana, DDS, $\mathrm{PhD}^{4}$; \\ Peter J. Polverini, DDS, DMSc ${ }^{5,6}$ \\ 1 Department of Oral and Maxillofacial Surgery, Indiana University Medical Center, Indianapolis, IN, USA \\ 2 Department of Pediatrics and Communicable Diseases, University of Michigan Medical School, Ann Arbor, MI, USA \\ 3 Department of Health Policy and Management, University of Michigan School of Public Health, Ann Arbor, MI, USA \\ 4 Department of Cariology, Restorative Sciences and Endodontics, University of Michigan School of Dentistry, Ann Arbor, MI, USA \\ 5 Department of Periodontics and Oral Medicine, University of Michigan School of Dentistry, Ann Arbor, MI, USA \\ 6 Department of Pathology, University of Michigan Medical School, Ann Arbor, MI, USA
}

Received: 4/26/2017; accepted: 2/2/2018.

doi: 10.1111/jphd.12267

Journal of Public Health Dentistry 78 (2018) 246-256

\begin{abstract}
Objectives: Collaborative and/or integrative care between oral health and primary care providers can increase access to care to a more expansive population, helping to mitigate oral health related disease. The objective of this review was to present and evaluate different types of care models that exist between oral health and primary care providers in pediatric settings.

Methods: A literature search was conducted using five databases: MEDLINE/ PubMed, ISI Web of Science, Dentistry and Oral Sciences Source, Cochrane Database, and EMBASE, to identify literature from January 1990 to January 2016. Combinations of controlled terms were utilized. Eligible sources targeted pediatric populations ages 1-17 and provided descriptions of existing collaborative and/or integrative models.

Results: Data related to the practice model, oral care provided, level of integration/ collaboration and workflow were extracted. Sixteen articles were included that discussed 24 models of collaboration. These models provided ranges of services, but each offered a minimum of oral health risk assessment, oral health instruction, topical fluoride application and assessment for further treatment. These models included different levels of collaboration based off a ranking system created by the authors with 16.6 percent (4) classified as low, 54.2 percent (13) as medium and 29.2 percent (7) as high.

Conclusions: Existing care models offered varying services and levels of integration and/or collaboration, but each offered a baseline of oral care. Most of these collaborations were based within Federally Qualified Health Centers and aimed to ease access to care issues.
\end{abstract}

\section{Introduction}

For much of the era of modern medicine, oral health has not been included in discussions of general health. There have been examples of pediatric dentists collaborating with medical colleagues to improve oral health for children, but this has been only a small segment of the population. Dental care was perceived by many as being elective care, and this showed in the education, practice, and financial realm of the health care delivery system, resulting in a system that failed to initiate preventive efforts for oral disease and ignored the links between oral disease and other health issues (1). Starting in the early 2000s, this view of oral health began to change. In the U.S. Department of Health and Human Services 2000 report, Oral Health in America, the lack of oral health care in the country was brought to the forefront of healthcare discussion (2). A National Call to Action to Promote Oral Health was 
introduced by the Surgeon General in 2003 to reinforce the idea that oral health was an integral part of systemic health and should be a focus of primary care practice and research (3). Most recently in 2014, the U.S. Department of HHS Health Resources and Services Administration introduced Integration of Oral Health and Primary Care Practice, a report that sought to improve early detection of oral health problems and preventive measures by increasing oral care proficiency among primary care physicians and encourage interdisciplinary integration and collaboration (4).

Due to efforts such as these, oral health has become an accepted part of general health - and for good reason. Research has shown that oral health plays a major role in determining overall systemic health in adults and children, but the implications for pediatric health are particularly concerning in contributing to childhood well-being and quality of life $(2,5)$. Poor oral health can lead to dental caries, the most common chronic disease in childhood, which in turn can lead to chronic tooth pain, resulting in more than 51 million hours of classroom time lost annually $(2,6,7)$. Dental caries can also have effects on chewing, swallowing, eating, and sleeping thereby posing problems with nutrition for proper development and health. These factors, along with the systemic issues that have shown links to poor oral health (diabetes, immune issues), may have long-term implications for pediatric patients. Preventative oral health care in children, then, is a crucial public health concern. The importance of an interdisciplinary healthcare system cannot be understated, and some success has been seen with overall dental disease rates having declined over the past three decades $(5,6)$. However, the positive results have not been shared equally among adult and pediatric patients, especially those from low socioeconomic and minority groups.

A systematic evaluation of the existing integrative and collaborative models between pediatric primary care and oral health providers has not been completed to the knowledge of the authors. Many sources explain existing models in small groups or isolates, but none have had the goal of exploring large numbers of these programs across the United States. That being said, it is difficult to determine whether this review is exhaustive. The aim of the present systematic literature review was to evaluate the types of integrative and collaborative care models in the US that exist between oral health providers and primary care practitioners in pediatric settings and to discern the implications of such models.

\section{Methods}

This systematic review was completed following the Preferred Reporting Items for Systematic Reviews and Meta-Analyses guidelines (8).

\section{Focused questions}

Do care models exist in the United States that incorporate primary care and oral health care for pediatric patients? If so, what types of models are available and what level of collaboration is present in these models?

\section{Search strategy}

A comprehensive literature search was conducted using the following databases in the review: Ovid MEDLINE/PubMed, ISI Web of Science, Dentistry and Oral Sciences Source, the Cochrane Database, and EMBASE, to identify literature from January 1990 up to January 2016 in the English language that described and evaluated different integrative and collaborative care models in the United States between pediatric health practitioners and oral health providers. Combinations of controlled terms (MeSH and EMTREE) and key words were utilized when available. Two reviewers (TG and PP) screened the reference list. The search terms are provided below:

MEDLINE/PubMed:

("comprehensive health care" [mesh] OR "models, organizational” [mesh] OR "comprehensive care model" [tiab] OR "comprehensive care models" [tiab] OR “collaborative care" [tiab] OR "collaborative practice" [tiab]) AND (pediatrics [mesh] OR pediatric [tiab] OR pediatrician [tiab] OR child health [tiab]) AND (dentistry[mesh] OR dental[tiab] OR dentistry[tiab] OR oral[tiab] OR dentist[tiab] OR "stomatognathic Diseases"[mesh]) AND english[la] ISI Web of Science:

TOPIC: (comprehensive care OR collaborative care) AND TOPIC: (pediatric OR paediatric OR child) AND TOPIC: (oral health or dental)

Dentistry and Oral Sciences Source:

("comprehensive care" OR "collaborative care") AND (pediatric* OR paediatric* OR child*) AND ("oral health" OR "dental”)

Cochrane Database:

(comprehensive care OR collaborative care) AND

(pediatric OR child) AND (oral health OR dental) EMBASE:

'organization and management'/de OR ((collaborative OR comprehensive) NEAR/5model $\left.{ }^{\star}\right):$ ab,ti AND ('pediatrics'/exp OR 'child'/exp OR pediatric*:ab,ti OR paediatric*:ab,ti OR child $^{\star}$ :ab,ti OR adolescent* ${ }^{\star}$ ab,ti) AND ('dentistry'/exp OR 'mouth disease'/exp OR dent $^{\star}: a b, t i$ OR oral health*:ab,ti) AND [english]/lim

Included and excluded papers and review articles were cross-referenced to locate additional publications. These articles were reviewed independently by the same two reviewers (TG and PP). A kappa value was calculated in order to express reviewer agreement concerning the included texts $(\kappa=0.95)$. 


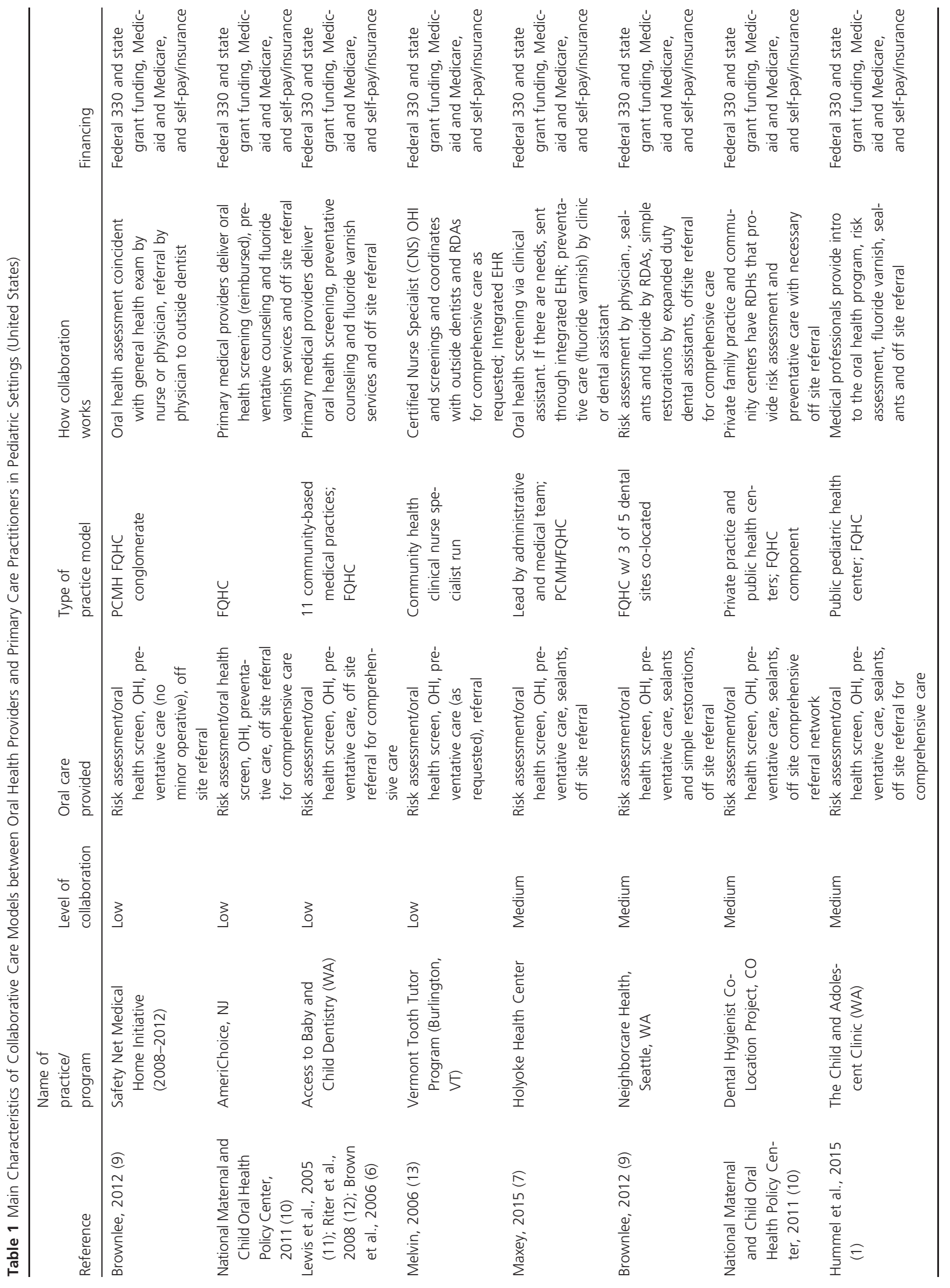




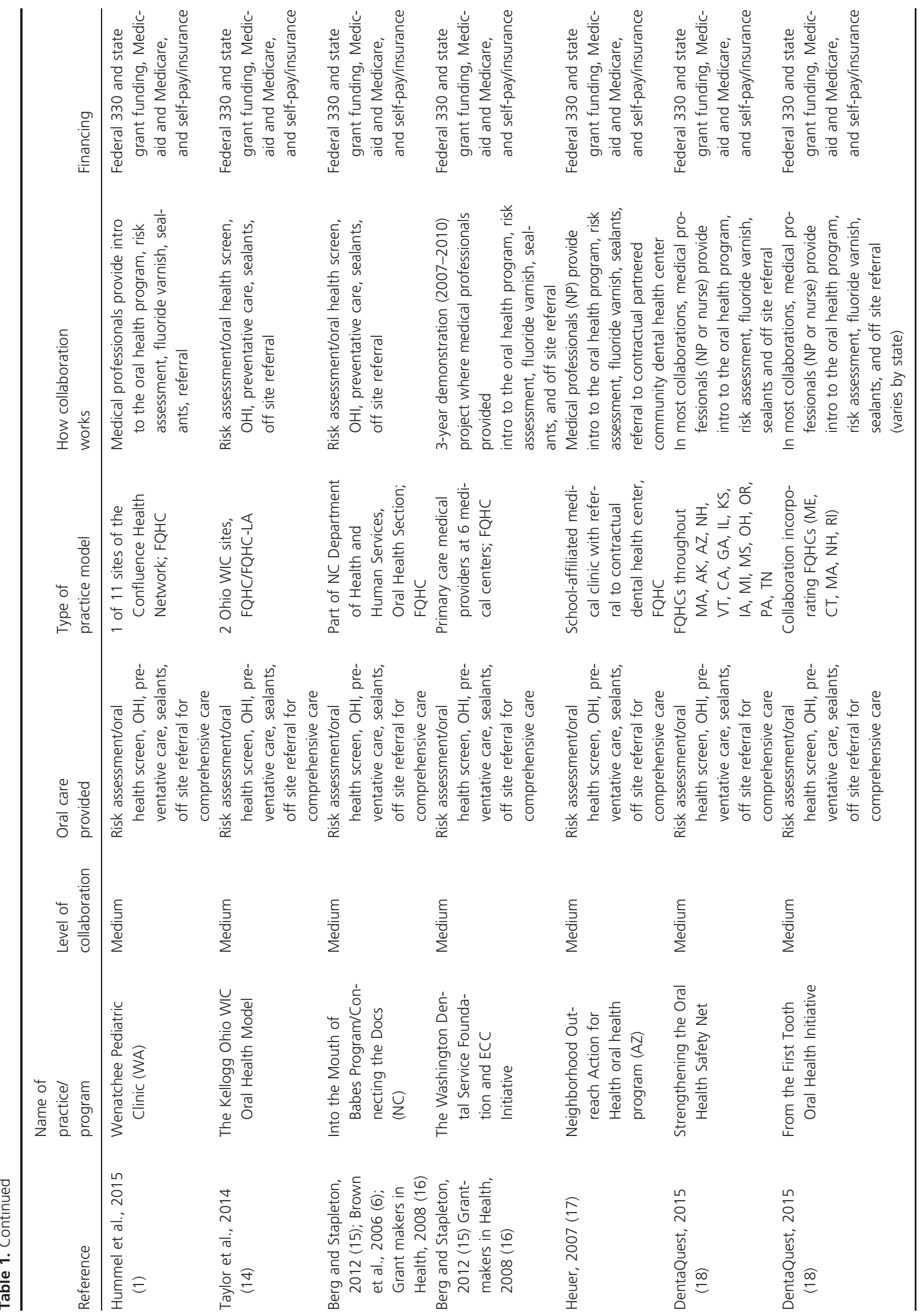




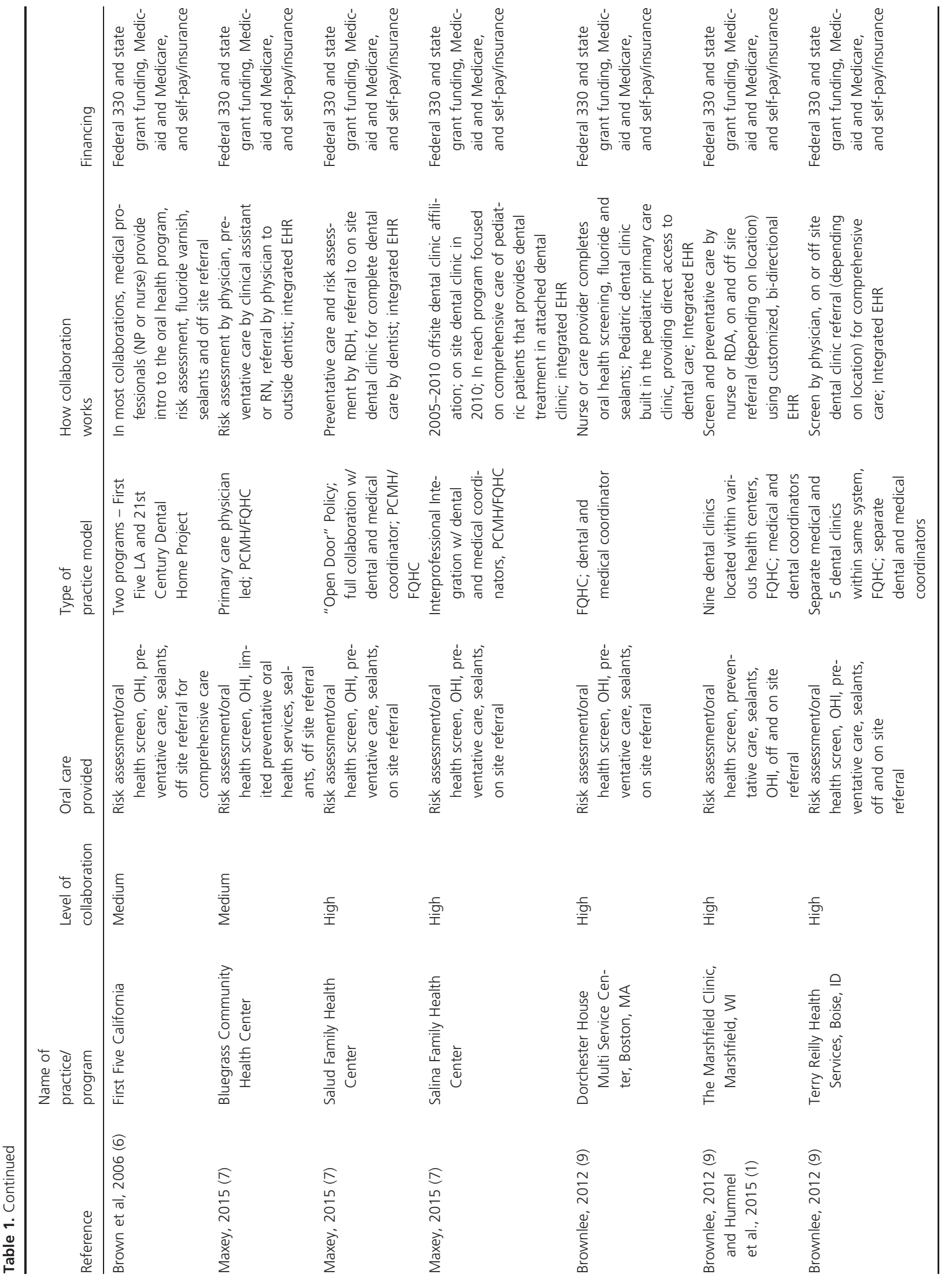




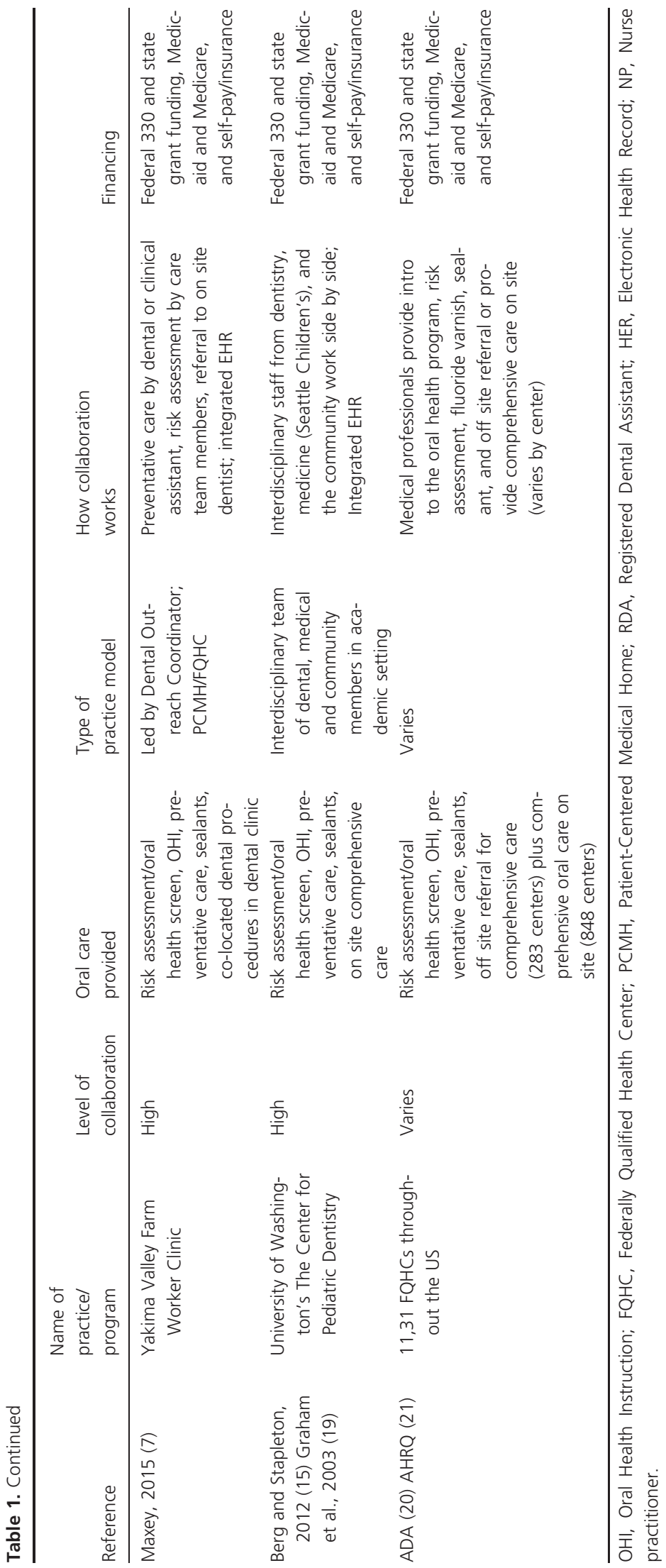




\section{Included elements}

Integrative and/or collaborative models involving pediatric health care and oral health care were included in the study. These models had to have a focus on preventive care, and either on site capabilities for comprehensive dental work or a specific system in place for referral of patients for comprehensive dental care. Two reviewers (TG and PP) made final decisions about inclusion of articles after full text assessment of possible relevant studies. Disagreements were resolved after collaborative discussion. The data extracted from each paper included the name of each practice or program and the following information for each: the level of integration and/or collaboration within each model, the oral care provided, the type of practice model and how the model worked in practice (financing, although not an analyzed criteria, was included in Table 1 for reference).

Each of the models was ranked concerning the level of integration and/or collaboration. The three available levels were low, medium, and high levels of integration/collaboration. A low level was defined as a model that provided a risk assessment/oral health screen, oral hygiene instruction (OHI), and preventive measures in the form of fluoride varnish on site, with necessary referrals to outside dental professionals for comprehensive dental care including dental radiographs, prophylaxis, and restorative care. A medium level was defined as all those measures provided by the low level model with the addition of radiographs, prophylaxis, and minor procedures on site such as sealant placement or minor operative procedures. A high level was defined as a model that provided all those measures provided by the medium level model with the addition of comprehensive dental treatment on site, whether that was through an indirect relationship via integrated electronic health record (EHR) at an attached dental clinic or direct collaboration within the same clinic (Table 2).

\section{Inclusion and exclusion criteria}

The sources were assessed based on the formulation of inclusion and exclusion criteria. Inclusion criteria included a) Pediatric patients, age range 1-17 years b) both dental and medical care provided c) preventative and routine dental care provided. The exclusion factors included: a) No focus on pediatric populations, age range $1-17$ years $(n=1)$, b) dental care described was in isolate and did not involve collaborative models with pediatric primary care physicians as defined by the authors $(n=24), c)$ focused on emergency dental care or dental trauma, not preventive or routine dental care $(n=6)$, d) medically oriented care was the focus, not a medicaldental collaboration $(n=2)$, and e) Focus on interprofessional education only, not including inter-professional care $(n=3)$.

\section{Results}

\section{Study selection}

The search strategy initially retrieved 1,494 potentially relevant citations, of which 83 were determined to be duplicates. An additional eight sources were obtained utilizing cross-reference mechanisms. After titles and abstracts were reviewed, 103 full text articles were assessed for eligibility. A total of 36 of these articles were excluded based upon the exclusion factors listed above. A total of 16 articles met these criteria and were included in this review, with a total of 24 models discussed (Table 1). A flow diagram was created to summarize the process of study selection (Figure 1).

\section{Collaboration features}

The main characteristics of the collaborations within the articles included in the systematic review were summarized in Table 1. All of the models were in the framework of Federally Qualified Health Centers (FQHC) or Federally Qualified Health Center Look-Alikes (FQHC-LA, which for the purposes of this analysis were considered FQHCs), except for two of the models, which were located in a university hospital $(15,19)$ and an elementary school nursing care facility $(13)$. One collaborative program was located in both an FQHC and a private practice establishment (10) Each care model was unique in terms of the type of practice model and how each one worked, but all of the models received both private and public funds and incorporated at least four primary services to its patients: Assessment of oral health risk via health screen, oral health instruction/anticipatory guidance to parents, topical fluoride application, and assessment for

Table 2 Description of the Levels of Collaboration Assigned to Each Collaborative Care Model within the Review

\begin{tabular}{ll}
\hline Description of levels of collaboration \\
\hline Level & Description \\
\hline Low & $\begin{array}{l}\text { Risk assessment/oral health screen, oral hygiene instruction, preventative care (fluoride varnish), off site referral for compre- } \\
\text { hensive care }\end{array}$ \\
Redium & $\begin{array}{l}\text { Risk assessment/oral health screen, oral hygiene instruction, preventative care (fluoride varnish), minor on site procedures, } \\
\text { including sealant placement and/or minor operative procedures, off site referral for comprehensive care }\end{array}$ \\
Righ & $\begin{array}{l}\text { Risk assessment/oral health screen, oral hygiene instruction, preventative care (fluoride varnish), comprehensive care pro- } \\
\text { vided on site }\end{array}$ \\
\hline
\end{tabular}




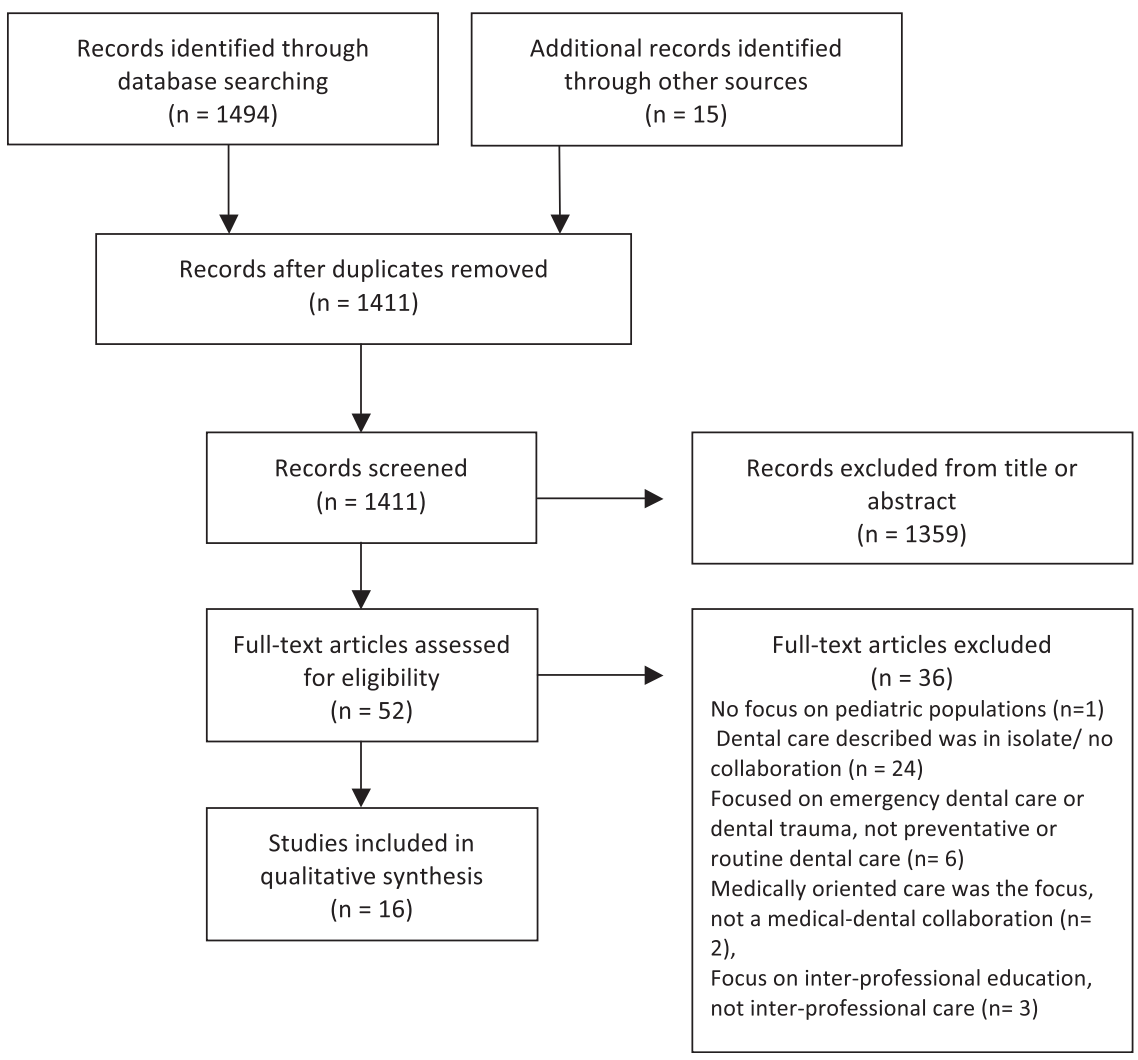

Figure 1 PRISMA (Preferred reporting items for systematic reviews and meta-analyses) flowchart of study selection process (8).

further treatment. Based on the ranking system previously noted, a total of four models were determined to be of low integration/collaboration (16.6 percent) $(6,9,10,12,13,22)$, thirteen models of a medium integration/collaboration (54.2 percent) $(1,6,7,9,10,14,16-18,23)$, and seven models of a high level of integration/collaboration (29.2 percent) $(1,7,9,15,19)$. A last group that was included in the review, which represents the 1,131 FQHCs that provide both medical and dental care, was of varied collaborative levels ranging from medium to high (20,21). Eight hundred forty-eight of all FQHCs (75 percent) offered the minimum level of oral health care established for this review in addition to either off sire referral for comprehensive care or on site restorative care via federal approval for supplementary health services $(20,24)$.

The main strategy for successful implementation of oral health and primary care collaboration has been through health centers. As a result, there is an entry at the end of table that encompasses all FQHCs as a whole. The analysis revealed that most of the successful high-level collaborations have commonalities. Each of these models used an integrated EHR shared by the medical and oral health professionals, which highlighted the importance of organized and detailed records for collaborative endeavors involving dental and medical providers. Although the low and medium level collaborations incorporated a referral system and some did use an integrated EHR, most of these collaborations did not specify the use of an integrated system. The majority of the high-level collaborations had individual dental and medical coordinators who had strong organizational leadership skills, which allowed the delegation of clinical and administrative tasks to motivated staff, while the lower level collaborations did not have these specific roles being fulfilled. The importance of careful organization through a focus on individual roles within the larger system was key to implementing a high functioning integrative/collaborative model. Incentives based on production and quality of care was vital to success as well. Future research should be assessed concerning funding and how it may be a determinant of the levels of collaboration and care provided within these collaborations.

\section{Discussion}

The aim of this systematic review was to provide examples of collaborative care models that exist in the United States and to analyze those models in terms of the level of integration/ collaboration, the type of practice model, and how the model works in an effort to increase access to dental care. This 
discussion will explain why these collaborations are important for the future of oral health in America and how they can be improved:

\section{Many people benefit}

The people that these models tend to serve are those who need care the most, as the majority of models were located within the FQHC framework. Based on the findings in this research, collaborations are a fairly new phenomenon, mirroring the increased focus on oral health discussed earlier. The infancy of this trend explains why there are no prototypical models of collaboration to base new collaborations on. Thus, there are varied models throughout the country. An intriguing find, then, was the number of medium and high levels of collaborations. With the target population, the importance of these models cannot be understated. In 2011, 4.4 million people utilized FQHCs for dental care alone. Out of those patients, approximately 33 percent were children 18 and younger (20). The fact that the majority of these models provided a variety of preventative care and even comprehensive oral care is reason to be optimistic about the future of oral health care for children who utilize these centers, which will be important in combating childhood and adolescent caries prevalence and incidence.

These models have provided an avenue for expanded care for those in need because all accept Medicaid and CHIP insurance plans. These insurance plans are not accepted at the majority of private practice offices. The decision to accept these insurances remains with the private provider and in many cases reimbursement levels dissuade providers from accepting patients with these insurances or only with the stipulation that they pay out of pocket for procedures. Medicaid and CHIP must be accepted at these locations, providing a venue for these patients to receive care. In addition, many of these collaborations are located in Health Professions Shortage Areas, further demonstrating how more children have increased opportunities to obtain the care that they need.

\section{Progress in oral health is being made in part because of these models}

The efforts that have been taken within the last decade to prioritize oral health can be seen in the decrease in the percentage of persons aged 5-19 with untreated dental caries, which has reached the goal values for Healthy People 2020 Project in terms of oral health objectives (3). These collaborations have played a role in decreasing oral disease among these pediatric populations and have provided a valuable asset in prioritizing oral health.

\section{Ideas for why these models work}

Because a large number of children receive exposure to medical care but not necessarily dental care at an early age, primary care practitioners are in a unique position to determine how these patients can best access recommended oral health care (25). In addition, parents tend to trust primary care physicians and adhere to the recommendations for their children - when health providers recommend visits to a dental professional, there tends to be an increase in dental visits among these same children $(26,27)$. Not only do pediatric primary care providers have the opportunity to see their patients on a more frequent basis, they also have the influence necessary to initiate oral health. This is why integration and collaboration between pediatric primary care practitioners and dental providers should be a part of the solution to access to care issues in terms of oral health. Partnerships between health professions can streamline necessary care and help to assure there are less roadblocks to provision of care by each entity playing active roles in the complete healthcare of the child.

\section{Barriers to formation}

Primary care physicians agree with the importance of increasing access to oral health care within their practices and realize their role in identifying dental problems and providing preventative care information to families $(22,28,29)$. In addition, they are able to "achieve an adequate level of accuracy" in designating children with carious lesions and noting those that need referral $(27,28,30)$. However, the problem is multifactorial. In two studies, time and reimbursement were main reasons why physicians did not examine oral health $(28,29)$. Reimbursement is a key issue, as dental and medical treatment is billed in different ways. There is a need for "diagnostic codes, uniform risk factors, and the ability for medical services to charge an additional fee for oral screening" $(9,31,32)$. These individuals or groups would be able to charge for a basic oral screening and increase the likelihood that these would be provided services. Another barrier concerns the funding and sustainability of such programs. Sustainability has been a problem for clinics when trying to implement these models, both in terms of financing and the retainment of patients. The authors acknowledge the issues with financing models and necessity of competitive state and federal grants, but these were not the focus of the review. Future studies will be focused on financing and its affect on the formation of these models. Add in the politicized policy process, including disagreements between the professions $(23,33)$, lack of educational models utilizing integrative/ collaborative practice $(15,16,33)$, and wide variations in regulation and infrastructure that makes coordination of care and patient retention difficult and it is clear that these structural barriers have prevented the formation of more of these models. 


\section{The changing landscape of the health professions}

Health professional school curriculums are beginning to put increased emphasis on inter-professional education and are helping aid cultural issues associated with these collaborative care models $(23,34)$. In addition, regulatory issues are becoming more streamlined since the implementation of integrated EHRs, as demonstrated by all the models in this review. The opportunity to create such models is greater than ever before.

\section{Note on quality assessment/risk of bias}

The results of this review should be considered mostly as a descriptive tool. The idea of integrative/collaborative care models originated in the early 2000s and has only recently gained traction in the health care field due to the Affordable Care Act $(10,35)$. Because of this fact, there is no analysis of how different models compare directly to others, nor how well the care provided by such models compares to private practice or solely individualized models. Clinical trials and surveys have been completed concerning how well primary care providers identify caries risk as compared to oral health professionals $(27,28)$ and dental professional opinions on these care efforts $(36,37)$, but nothing has been completed concerning outcomes of care compared to traditional dental settings. There is such variance between different oral care providers that this would be an almost impossible endeavor. The authors acknowledge that there are many more efforts to promote oral health in nontraditional venues across the United States, but the specific inclusion factors eliminated many of these models from this review.

\section{Conclusions}

Integrative and/or models allow the opportunity to provide preventative oral care to the most vulnerable populations. These models, then, have the potential to combat oral disease in America. One of the ways to ensure that enough is being done to expand access to dental care, increase dental visits, and help mitigate the problem of childhood caries is to expand partnerships between primary care providers and oral health providers. These partnerships have started to develop across the country since oral health became an accepted and vital sector of the health care system as a whole. There are still barriers that exist that discourage the formation of integrative and/or collaborative efforts. However, recent efforts to increase the efficiency of and streamline the health care system provide intriguing opportunities to eliminate those barriers and encourage more collaborative work between the professions.

\section{References}

1. Hummel J, Phillips KE, Holt B, Hayes C. Oral health: an essential component of primary care [white paper]. Seattle, WA: Qualis Health for the National Interprofessional Initiative on Oral Health; 2015.

2. U.S. Department of Health and Human Services, Public Health Service, Office of the Surgeon General. Oral health in America: a report of the surgeon general. Rockville, MD: National Institutes of Health, National Institute of Dental and Craniofacial Research; 2000.

3. US Department of Health and Human Services, Public Health Service, Centers of Disease Control and Prevention. A national call to action to promote oral health. Rockville, MD: National Institutes of Health, National Institute of Dental and Craniofacial Research; 2003.

4. U.S. Department of Health and Human Services, Public Health Service, Office of the Surgeon General. Integration of oral health and primary care practice. Rockville, MD: National Institutes of Health, National Institute of Dental and Craniofacial Research; 2014.

5. Filstrup FL, Briskie D, da Fonseca M, Lawrence L, Wandera A, Inglehart MR. Early childhood caries and quality of life: child and parent perspectives. Pediatr Dent. 2003;25(5):431-40.

6. Brown A, Lowe E, Zimmerman B, Crall JJ, Foley M, Nehring M. Preventing early childhood caries: lessons from the field. Pediat Dent. 2006;28(6):553-60.

7. Maxey H. Integration of oral health with primary care in health centers: profiles of five innovative models [white paper]. Bethesda, MD: National Association of Community Health Centers; 2005.

8. Liberati A, Altman DG, Tetzlaff J, Mulrow C, Gøtzsche PC, Ioannidis JP, Clarke M, Devereaux PJ, Kleijnen J, Moher D. The PRISMA statement for reporting systematic reviews and meta-analyses of studies that evaluate health care interventions: explanation and elaboration. PLoS Med 2009; 6(7):e1000100.

9. Brownlee B. Oral health integration in the patient-centered medical home (PCMH) environment: case studies from community health centers [white paper]. Seattle, WA: Qualis Health for the Dentaquest Foundation Qualis; 2012.

10. National Maternal and Child Oral Health Policy Center. Children's oral health in the health home. 2011 [cited 2016 Jan 23]. Available from: http://nmcohpc.net/resources/ TrendNotes_May_2011.pdf

11. Lewis C, Lynch H, Richardson L. Fluoride varnish use in primary care: what do providers think? Pediatrics. 2005; 115(1):e69-76.

12. Riter D, Maier R, Grossman DC. Delivering preventive oral health services in pediatric primary care: a case study. Health Aff (Millwood). 2008;27(6):1728-32.

13. Melvin CS. A collaborative community-based oral care program for school-age children. Clin Nurse Spec. 2006; 20(1):18-22. 
14. Taylor E, Marino D, Thacker S, DiMarco M, Huff M, Biordi D. Expanding oral health preventative services for young children: a successful interprofessional model. J Allied Health. 2014;43(1):e5-9.

15. Berg JH, Stapleton FB. Physician and dentist: new initiatives to jointly mitigate early childhood oral disease. Clin Pediatr. 2012;51(6):531-7.

16. Critical services for our children: Integrating mental and oral health into primary care. Issue Brief (Grantmakers Health). 2008;(30):1-27; i-iii.

17. Heuer $S$. Integrated medical and dental health in primary care. J Spec Pediatr Nurs. 2007;12(1):61-5.

18. DentaQuest Foundation. Strengthening the Oral Health Safety Net. Programs and partnerships. 2015 [cited 2016 Mar 4]. Available from: http://dentaquestfoundation.org/ programs-partnerships/national-partnerships/strengtheningoral-health-safety-net

19. Graham E, Negron R, Domoto P, Milgrom P. Children's oral health in the medical curriculum: a collaborative intervention at a university-affiliated hospital. J Dent Educ. 2003;67(3):338-47.

20. American Dental Association. Federally qualified health centers FAQ. 2017 [cited 2016 Mar 3]. Available from: http:// www.ada.org/en/public-programs/action-for-dental-health/ access-to-care/federally-qualified-health-centers-faq

21. Agency for Healthcare Research and Quality. Defining the PCMH. U.S. Department of Health and Human Services; 2017 [cited 2016 Mar 4]. Available from: https://pcmh.ahrq. gov/page/defining-pcmh

22. Lewis C, Robertson AS, Phelps S. Unmet dental care needs among children with special health care needs: implications for the medical home. Pediatrics. 2005;116(3):E426-31.

23. Hallas D, Fernandez JB, Herman NG, Moursi A. Identification of pediatric oral health core competencies through interprofessional education and practice. Nurs Res Pract. 2015;2015:360523.

24. Dela Cruz GG, Rozie RG, Slade G. Dental screening and referral of young children by pediatric primary care providers. Pediatrics. 2004;114(5):e642-52.

25. Beil HA, Rozier RG. Primary health care providers' advice for a dental checkup and dental use in children. Pediatrics. 2010; 126(2):e435-41.

26. Pierce KM, Rozier RG, Vann WF Jr. Accuracy of pediatric primary care providers' screening and referral for early childhood caries. Pediatrics. 2002;109(5):E82.
27. Lewis CW, Grossman DC, Domoto PK, Deyo RA. The role of the pediatrician in the oral health of children: a national survey. Pediatrics. 2000;106(6):E84.

28. Roberts MW, Keels MA, Sharp MC, Lewis JL Jr. Fluoride supplement prescribing and dental referral patterns among academic pediatricians. Pediatrics. 1998; 101(1):E6.

29. Pahel BT, Rozier RG, Stearns SC. Agreement between structured checklists and medicaid claims for preventive dental visits in primary care medical offices. Health Informatics J. 2010;16(2):115-28.

30. Lewis CW, Boulter S, Keels MA, Krol DM, Mouradian WE, O'Connor KG, Quinonez RB. Oral health and pediatricians: results of a national survey. Acad Pediatr. 2009;9(6):457-61.

31. Jones RB. "Savage inequities": can public/private partnership impact oral health access in the United States? J Public Health Dent. 1998;58(1):2-6.

32. Dolce MC. Integrating oral health into professional nursing practice: an interprofessional faculty tool kit. J Prof Nurs. 2014;30(1):63-71.

33. Mertz E, Lindler V, Dower C. Collaborative practice in American dentistry: practice and potential. San Francisco, CA: Center for the Health Professions at the University of San Francisco, CA; 2011.

34. Reeves S, Perrier L, Goldman J, Freeth D, Zwarenstein M. Interprofessional education: effects on professional practice and healthcare outcomes (update). Cochrane Database Syst Rev. 2013;(3):CD00221.

35. Baker GH Jr. Integration of oral and general health in maternal and child populations. J Public Health Dent. 1990; 50(6 Spec No):402-5.

36. Minah G, Lin C, Coors S, Rambob I, Tinanoff N, Grossman LK. Evaluation of an early childhood caries prevention program at an urban pediatric clinic. Pediatr Dent. 2008; 30(6):499-504.

37. Quinonez RB, Kranz AM, Long M, Rozier RG. Care coordination among pediatricians and dentists: a crosssectional study of opinions of North Carolina dentists. BMC Oral Health. 2014;14:33.

38. Higgins JPT, Green S, editors. Cochrane handbook for systematic reviews of interventions (Version 5.1.0). The Cochrane Collaboration. 2011 [cited 2011 Mar]. Available from: www.cochrane-handbook.org 